\title{
Development of amoxicillin resistance in Escherichia coli after exposure to remnants of a non-related phagemid-containing $E$. coli: an exploratory study
}

Joep J. J. M. Stohr ${ }^{1,2^{*}}$, Marjolein F. Q. Kluytmans-van den Bergh ${ }^{1,3,4}$, Carlo J. M. M. Verhulst ${ }^{1}$, John W. A. Rossen ${ }^{5}$ and Jan A. J. W. Kluytmans ${ }^{1,2,3,4}$

\begin{abstract}
Objective: To determine the effect of exposure to remnants of a phagemid-containing $E$. coli, killed by treatment with a propanol-based hand rub, on antimicrobial resistance in E. coli isolates.

Methods: An in vitro model was developed in which a clinical E. coli isolate (EUR1) was exposed to remnants of an E. coli K-12 strain containing a phagemid (pBS-E12) strain treated with Sterillium ${ }^{\oplus}$. A series of 200 experiments was performed using this in vitro model. As a control, a series of 400 experiments was performed where the EUR1 was exposed either to the remnants of an E. coli K-12 strain (not containing a phagemid) (E12) treated with Sterillium ${ }^{\circledast}$ $(n=200)$ or to dried Sterillium ${ }^{\circledast}$ only $(n=200)$. The number of experiments that showed growth of an amoxicillinresistant EUR1 isolate was evaluated in all three groups. An additional 48 experiments were performed in which a different clinical E. coli isolate (EUR2) was exposed to remnants of the pBS-E12 treated with Sterillium ${ }^{\oplus}$. Wholegenome sequencing and phenotypic testing for AmpC beta-lactamase production was performed to investigate the mechanism behind this resistance development.

Results: In 22 (11.0\%) of 200 experiments in which the EUR1 isolate was exposed to remnants of a pBS-E12 an amoxicillin-resistant mutant isolate was obtained, as opposed to only $2(1.0 \%)$ of 200 experiments involving the exposure of the EUR1 to Sterillium ${ }^{\circledR}$ only (risk difference: 10.0\%; 95\% Cl 5.4-14.6\%)) and 1 (0.5\%) of 200 experiments involving the exposure of the EUR1 isolate to the remnants of the phagemid-free E12 (risk difference: 10.5\%; $95 \% \mathrm{Cl}$ 6.1-14.9\%). In 1 (2.1\%) of the 48 experiments in which the EUR2 isolate was exposed to remnants of a pBS-E12 an amoxicillin-resistant mutant isolate was obtained. The development of resistance in all experiments was due to mutations in the promoter/attenuator region of the chromosomal AmpC beta-lactamase (cAmpC) gene leading to cAmpC hyperproduction.

(Continued on next page)
\end{abstract}

\footnotetext{
* Correspondence: joep.stohr@gmail.com

'Department of Infection Control, Amphia Hospital, Breda, the Netherlands

'Laboratory for Medical Microbiology and Immunology,

Elisabeth-TweeSteden Hospital, Tilburg, the Netherlands

Full list of author information is available at the end of the article
}

(c) The Author(s). 2020 Open Access This article is licensed under a Creative Commons Attribution 4.0 International License, which permits use, sharing, adaptation, distribution and reproduction in any medium or format, as long as you give appropriate credit to the original author(s) and the source, provide a link to the Creative Commons licence, and indicate if changes were made. The images or other third party material in this article are included in the article's Creative Commons licence, unless indicated otherwise in a credit line to the material. If material is not included in the article's Creative Commons licence and your intended use is not permitted by statutory regulation or exceeds the permitted use, you will need to obtain permission directly from the copyright holder. To view a copy of this licence, visit http://creativecommons.org/licenses/by/4.0/ The Creative Commons Public Domain Dedication waiver (http://creativecommons.org/publicdomain/zero/1.0/) applies to the data made available in this article, unless otherwise stated in a credit line to the data. 
(Continued from previous page)

Conclusion: Exposure of an E. coli isolate to another phagemid-containing E. coli that was treated with propanolbased hand rub increased the development of amoxicillin resistance. Although phagemids are cloning vectors that are not present in clinical isolates, this finding may have implications for hand disinfection practices in healthcare facilities.

Keywords: Sterillium, AmpC, Antimicrobial resistance, Disinfection

\section{Introduction}

The last decades we have seen a dramatic worldwide increase in antimicrobial resistance (AMR) among Gram-negative bacteria. One of the most remarkable phenomena is the rapid increase of plasmid-mediated beta-lactam resistance in Escherichia coli [1-3]. International infection control guidelines recommend several measures to control the spread of AMR, among which propanol-based disinfection of hands is vital [4, 5]. These disinfection methods rapidly and effectively decrease the number of viable bacteria on hands and thereby limiting the spread of resistant bacteria and healthcare-related infections [6-9]. However, intact bacterial DNA could potentially persist after propanol-based bacterial cell lysis and mechanical cleaning and serve as a source of resistance determinants for other bacteria that reach the disinfected area $[10,11]$. Uptake of plasmid and chromosomal bacterial DNA from the environment through natural transformation has already been described as a method of resistance acquisition in streptococci, Helicobacter spp. and various other bacteria [12-16]. Recent studies have also shown that E. coli is able to take up DNA in various environments [16-21]. However, it remains unclear to what extent the uptake of resistance plasmids via natural transformation contributes to the development of AMR in E. coli. Moreover, it is unknown whether exposure to environmental DNA remainders (plasmids) could facilitate this form of AMR acquisition. Therefore, we developed an in vitro model in which a clinical E. coli isolate (EUR1) was exposed to a pBleuscript $\mathrm{KS}(-)$ phagemid (encoding an amoxicillin resistance gene)-containing $E$. coli $\mathrm{K}-12$ strain which had been treated with alcohol (Sterillium ${ }^{\circ}$ ). In this experiment, we did not observe plasmid transfer (results on file), yet we did observe the development of beta-lactam resistance in the EUR1 isolate. This observation led to the hypothesis that exposure to remnants of phagemid-containing $E$. coli (killed by treatment with Sterillium ${ }^{\circ}$ ) could lead to the development of AMR in $E$. coli isolates that came in contact with the remnants of the killed E. coli $\mathrm{K} 12$ strain, through increased chromosomal mutations. Although not previously reported for E. coli, studies have already shown that in Salmonella spp. and Pseudomonas spp. the presence of external DNA could lead to an increase in the development of
AMR through mechanisms other than transformation $[22,23]$. In this exploratory study, we used an in vitro model to compare the rate at which amoxicillin-resistant mutants developed for an E. coli isolate exposed to amoxicillin and remnants of a pBleuscript KS(-) phagemid containing E. coli $\mathrm{K}-12$ strain treated with Sterillium ${ }^{\circ}$, amoxicillin and remnants of a phagemid-free K-12 strain treated with Sterillium ${ }^{\circ}$ or amoxicillin and dried Sterillium ${ }^{\bullet}$ only.

\section{Method \\ Isolate selection}

An E. coli -K12 (JM83, ATCC $35607^{\text {tri }}$ ) harbouring a IIpBleuscript KS (-) phagemid (ATCC ${ }^{\bullet} 87047^{\mathrm{mm}}$ ) (copy number: 300-500) containing an amoxicillin-resistance gene $\left(b l a_{\text {TEM-116 }}\right)$ (pBS-E12), was chosen to be exposed to the propanol-based hand rub and two E. coli isolates (EUR1 and EUR2) obtained from routine clinical cultures (EUR1: peritoneal fluid culture, EUR2: urine culture) of epidemiologically unrelated patients were selected to be exposed to remnants of the pBS-E12, Sterillium ${ }^{\circ}$ and amoxicillin (for mutant selection). Strain characteristics of the used strains are depicted in Supplementary table S1. Features of the high copy number pBleuscript phagemid are depicted in Supplementary table S2. The selection of the EUR1 and EUR2 was based on antimicrobial susceptibility pattern, i.e. susceptible to amoxicillin and resistant to trimethoprim based on EUCAST clinical breakpoints version 9.0 [24]. The pBSE12 has a trimethoprim MIC below the clinical breakpoint. Antimicrobial susceptibility testing was performed using the VITEK $2^{\circ}$ system (bioMérieux, Marcy l'Etoile, France). The minimal inhibitory concentration (MIC) for amoxicillin was additionally tested with ETEST ${ }^{\circ}$ (bioMérieux, Marcy l'Etoile, France). The MICs of the EUR1, EUR2, and pBS-E12 for the various tested antimicrobial agents are shown in Table 1 .

\section{Exposure to propanol-based hand rub (Sterillium $\left.{ }^{\circledR}\right)$ and resistance induction}

Sterile glass surfaces $(25 \mathrm{mmx} 18 \mathrm{~mm})$ were inoculated with $10 \mu \mathrm{L}$ of a $0.5 \mathrm{McFarland}$ suspension of the pBSE12 isolate $\left(1.5 \times 10^{6}\right.$ colony forming units $\left.(\mathrm{CFU})\right)$ in brain-heart infusion broth (BHI). The inoculation of the glasses was performed in a Kojair biosafety cabinet class 
Table 1 Minimal inhibitory concentration for various antibiotics

\begin{tabular}{|c|c|c|c|c|c|c|c|c|}
\hline \multirow[t]{3}{*}{ Isolate } & \multicolumn{7}{|c|}{$\mathrm{MIC}(\mathrm{mg} / \mathrm{L})$} & \multirow{3}{*}{$\begin{array}{l}\text { D68C AmpC \& ESBL } \\
\text { Detection set conclusion } \\
\text { AmpC hyperproduction } ¥\end{array}$} \\
\hline & \multirow{2}{*}{$\begin{array}{l}\text { E-test }^{\oplus} \\
\text { amox }\end{array}$} & \multicolumn{6}{|l|}{ Vitek $^{\otimes}$} & \\
\hline & & amcl & pita & cfrx & cfxt & $\mathrm{cftz}$ & $\mathrm{cftx}$ & \\
\hline pBS-E12 & $>=256$ & $>=32$ & $>=128$ & 8 & $<=4$ & $<=1$ & $<=1$ & No \\
\hline EUR1 & 6 & $<=2$ & $<=4$ & 4 & $<=4$ & $<=1$ & $<=1$ & Yes \\
\hline EUR1M1 & $>=256$ & $>=32$ & 8 & 32 & $>=64$ & 4 & $<=1$ & Yes \\
\hline EUR1M2 & $>=256$ & $>=32$ & 16 & 32 & $>=64$ & 4 & $<=1$ & Yes \\
\hline EUR1M3 & $>=256$ & $>=32$ & $<=4$ & 16 & 16 & $<=1$ & $<=1$ & Yes \\
\hline EUR1M4 & $>=256$ & $>=32$ & 8 & 32 & 32 & $<=1$ & $<=1$ & Yes \\
\hline EUR1M5 & $>=256$ & $>=32$ & 8 & $>=64$ & $>=64$ & 4 & 2 & Yes \\
\hline EUR1M6 & 256 & $>=32$ & $<=4$ & 16 & $>=64$ & $<=1$ & $<=1$ & Yes \\
\hline EUR1M7 & $>=256$ & $>=32$ & 8 & 32 & $>=64$ & 4 & $<=1$ & Yes \\
\hline EUR1M8 & $>=256$ & $>=32$ & $<=4$ & 16 & 16 & $<=1$ & $<=1$ & Yes \\
\hline EUR1M9 & 256 & $>=32$ & $<=4$ & 16 & 16 & $<=1$ & $<=1$ & Yes \\
\hline EUR1M10 & 128 & $>=32$ & $<=4$ & 16 & 32 & $<=1$ & $<=1$ & Yes \\
\hline EUR1M11 & 128 & $>=32$ & $<=4$ & 32 & 16 & $<=0.25$ & $<=0.25$ & Yes \\
\hline EUR1M12 & $>=256$ & $>=32$ & 16 & 32 & 16 & 2 & 1 & Yes \\
\hline EUR1M13 & $>=256$ & $>=32$ & 16 & 32 & 32 & 2 & 1 & Yes \\
\hline EUR1M14 & $>=256$ & $>=32$ & 16 & 32 & 16 & 1 & $<=0.25$ & Yes \\
\hline EUR1M15 & 196 & $>=32$ & $<=4$ & 16 & 16 & 0.5 & $<=0.25$ & Yes \\
\hline EUR1M16 & 196 & $>=32$ & $<=4$ & 16 & 16 & 0.5 & $<=0.25$ & Yes \\
\hline EUR1M17 & 256 & $>=32$ & $<=4$ & 32 & 32 & 2 & 0.5 & Yes \\
\hline EUR1M18 & $>=256$ & $>=32$ & 16 & 32 & 16 & 2 & 1 & Yes \\
\hline EUR1M19 & $>=256$ & $>=32$ & 8 & 32 & 16 & 2 & $<=0.25$ & Yes \\
\hline EUR1M20 & 64 & 16 & $<=4$ & 4 & $<=4$ & 0.5 & $<=0.25$ & Yes \\
\hline EUR1M21 & $>=256$ & $>=32$ & 16 & 32 & 16 & 4 & 1 & Yes \\
\hline EUR1M22 & 256 & $>=32$ & 16 & 32 & 16 & 4 & 1 & Yes \\
\hline EUR1S1 & $>=256$ & $>=32$ & $<=4$ & 16 & 32 & $<=1$ & $<=1$ & Yes \\
\hline EUR1S2 & $>=256$ & $>=32$ & 8 & 32 & $>=64$ & 2 & $<=1$ & Yes \\
\hline EUR1E1 & $>=256$ & $>=32$ & $<=4$ & 16 & 32 & $<=1$ & $<=1$ & Yes \\
\hline EUR2 & 1 & $<=2$ & $<=4$ & 2 & $<=4$ & $<=1$ & $<=1$ & No \\
\hline EUR2M1 & $>=256$ & $>=32$ & 8 & 8 & $<=4$ & $<=1$ & $<=1$ & Yes \\
\hline
\end{tabular}

MICs were measured with ETEST ${ }^{\oplus}$ (bioMérieux, Marcy l'Etoile, France) (amoxicillin) or the VITEK $2^{\circledast}$ system (bioMérieux, Marcy l'Etoile, France). ¥Interpretation of D68C AmpC \& ESBL Detection set according to manufacturers instruction. Zone diameters as measured in the D68C AmpC \& ESBL Detection set are depicted in Supplementary table S3. amox: amoxicillin; amcl: amoxicillin-clavulanic acid; pita: piperacillin-tazobactam; cfrx: cefuroxime; cfxt: cefoxitin; cftz: ceftazidime; cftx: cefotaxime; mero: meropenem; imip: imipenem;M: amoxicillin-resistant mutant after exposure to remnants of the pBS-E12 and Sterillium ${ }^{\circledR}$ S: amoxicillin-resistant mutant after exposure to Sterillium ${ }^{\oplus}$ onlyE: amoxicillin-resistant mutant after exposure to E12 and Sterillium ${ }^{\oplus}$

II Silver Line. The inoculated glasses were left to dry at ambient air temperature for $10+/-1 \mathrm{~min}$. Subsequently, the glasses were inoculated with $30 \mu \mathrm{L}$ of Sterillium ${ }^{\oplus}$, which was spread across the entire glass surface using sterile plastic sticks. The Sterillium ${ }^{\bullet}$ used contained per $100 \mathrm{~g}$ of solution: Propan-2-ol $45.0 \mathrm{~g}$, propan-1-ol $30.0 \mathrm{~g}$ and mecetronium etilsulfate $0.2 \mathrm{~g}$. The ratio of the volume of inoculated Sterillium ${ }^{\circ}$ to glass surface area $\left(3 \times 10^{-5} \mathrm{~L}\right.$ : $0.00045 \mathrm{~m}^{2}$ ) was chosen to reflect the ratio of the volume of Sterillium ${ }^{\odot}$ used in hand disinfection to the average hand surface area $\left(3 \times 10^{-3} \mathrm{~L}: 0.045 \mathrm{~m}^{2}\right)[25,26]$. The
PBS-E12 and Sterillium ${ }^{\oplus}$-containing glasses were left to dry at ambient room temperature for $10+/-1 \mathrm{~min}$. Subsequently, the glasses were inoculated with $10 \mu \mathrm{L}$ of an 0.5 McFarland suspension of the EUR1 isolate $\left(1.5 \times 10^{6}\right.$ CFU) in BHI, which was spread across the entire glass surface using sterile plastic sticks. The re-inoculated glasses were left to dry at ambient air temperature for 10 $+/-1$ min. Subsequently, the glasses were placed in a container with $4 \mathrm{~mL}$ of $\mathrm{BHI}$, containing $1 \mathrm{mg} / \mathrm{L}$ amoxicillin, vortexed for $30 \mathrm{~s}$ at 2800 rotations per minute and incubated at 35 to $37 \mathrm{C}^{\circ}$. The duration the isolates were 
subjected to a subinhibitory amoxicillin concentration is meant to represent the duration of subinhibitory amoxicillin plasma-concentrations in patients starting amoxicillin treatment [27]. After $1 \mathrm{~h}$ of incubation, an additional $1 \mathrm{~mL}$ of BHI broth containing $320 \mathrm{mg} / \mathrm{L}$ amoxicillin was added to the glasses carrying containers, resulting in $5 \mathrm{~mL}$ of BHI broth containing $64.8 \mathrm{mg} / \mathrm{L}$ amoxicillin in the glasses carrying containers. The containers were then incubated at 35 to $37 \mathrm{C}^{\circ}$ for $72 \mathrm{~h}$ and visually inspected for bacterial growth at 8, 24, 48 and $72 \mathrm{~h}$. At visible growth or after 72 $h$ of incubation in absence of visible growth, $1 \mu \mathrm{L}$ of the suspension was plated on a Muller Hinton agar containing $8 \mathrm{mg} / \mathrm{L}$ trimethoprim and $64 \mathrm{mg} / \mathrm{L}$ amoxicillin (MH-TA). The inoculated MH-TA plates were incubated for 18 to $24 \mathrm{~h}$ at 35 to $37 \mathrm{C}^{\circ}$. Colonies growing on the MH-TA underwent species identification performed with VITEK MS (bioMérieux, Marcy l'Etoile, France), using the VITEK MS Knowledge Base v.2.0, and antimicrobial susceptibility testing performed with the VITEK $2^{\circ}$ system (bioMérieux, Marcy l'Etoile, France), using EUCAST susceptibility breakpoints version 9.1 [24]. The MIC of all isolates to amoxicillin was additionally tested using ETEST $^{\circ}$ (bioMérieux, Marcy l'Etoile, France). Grown E. coli colonies with a MIC of $>8 \mathrm{mg} / \mathrm{L}$ for trimethoprim, measured in the VITEK $2^{\circ}$ system (bioMérieux, Marcy l'Etoile, France), and with a MIC of $>64 \mathrm{mg} / \mathrm{L}$ for amoxicillin, measured with $\mathrm{ETEST}^{\bullet}$ (bioMérieux, Marcy l'Etoile, France), were considered mutants. A series of 200 experiments were performed using the EUR1 isolate. Additionally, a series of 48 experiments were performed using the EUR2 isolate.
Experiments with the EUR1 and EUR2 isolates were performed on separate days. For each experiment colonies were picked of the EUR1 and EUR2 isolate and the pBSE12 strain in stationary growth phase after incubation at 35 to $37 \mathrm{C}^{\circ}$ for 18 to $24 \mathrm{~h}$ on Muller Hinton agar. The pBS-E12 strain was inoculated on Muller Hinton agar plates containing an amoxicillin $10 \mu \mathrm{g}$ disk. Only colonies of the pBS-E12 growing in the direct surrounding of the amoxicillin disk were used in the experiments. The experimental setup is illustrated in Fig. 1.

\section{Control experiments}

A series of 200 control experiments were performed in which the glasses were only inoculated with Sterillium and the EUR1 and a series of 200 experiments were performed in which the glasses were inoculated with the E. coli K-12 strain (JM109, ATCC $53323^{\text {tm }}$ ) not containing the pBleuscript KS (-) high copy number phagemid (E12) (Supplementary Table S1), Sterillium ${ }^{\circ}$ and the EUR1 isolate. Experiments were performed on separate days. For each experiment colonies were picked of the EUR1 isolate and E12 strain after incubation at 35 to $37 \mathrm{C}^{\circ}$ for 18 to $24 \mathrm{~h}$ on Muller Hinton agar. The setup of the control experiments is illustrated in Fig. 2.

\section{Disinfection experiments}

To determine if the pBS-E12 was still culturable after exposure to Sterillium ${ }^{\circ}$, we performed a series of 100 experiments in which the glasses were inoculated with the pBS-E12 followed by Sterillium ${ }^{\circ}$ only. The inoculated

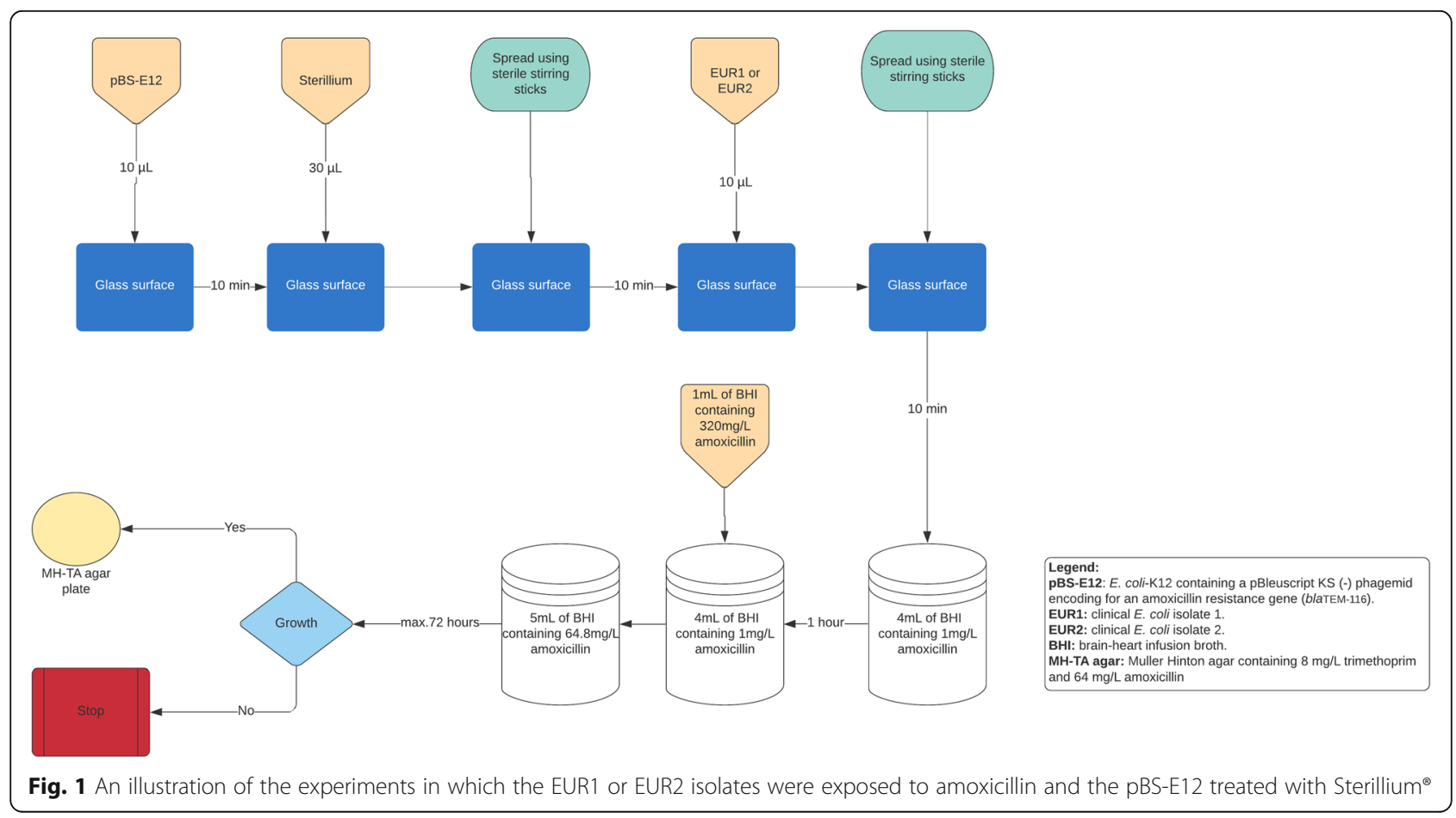




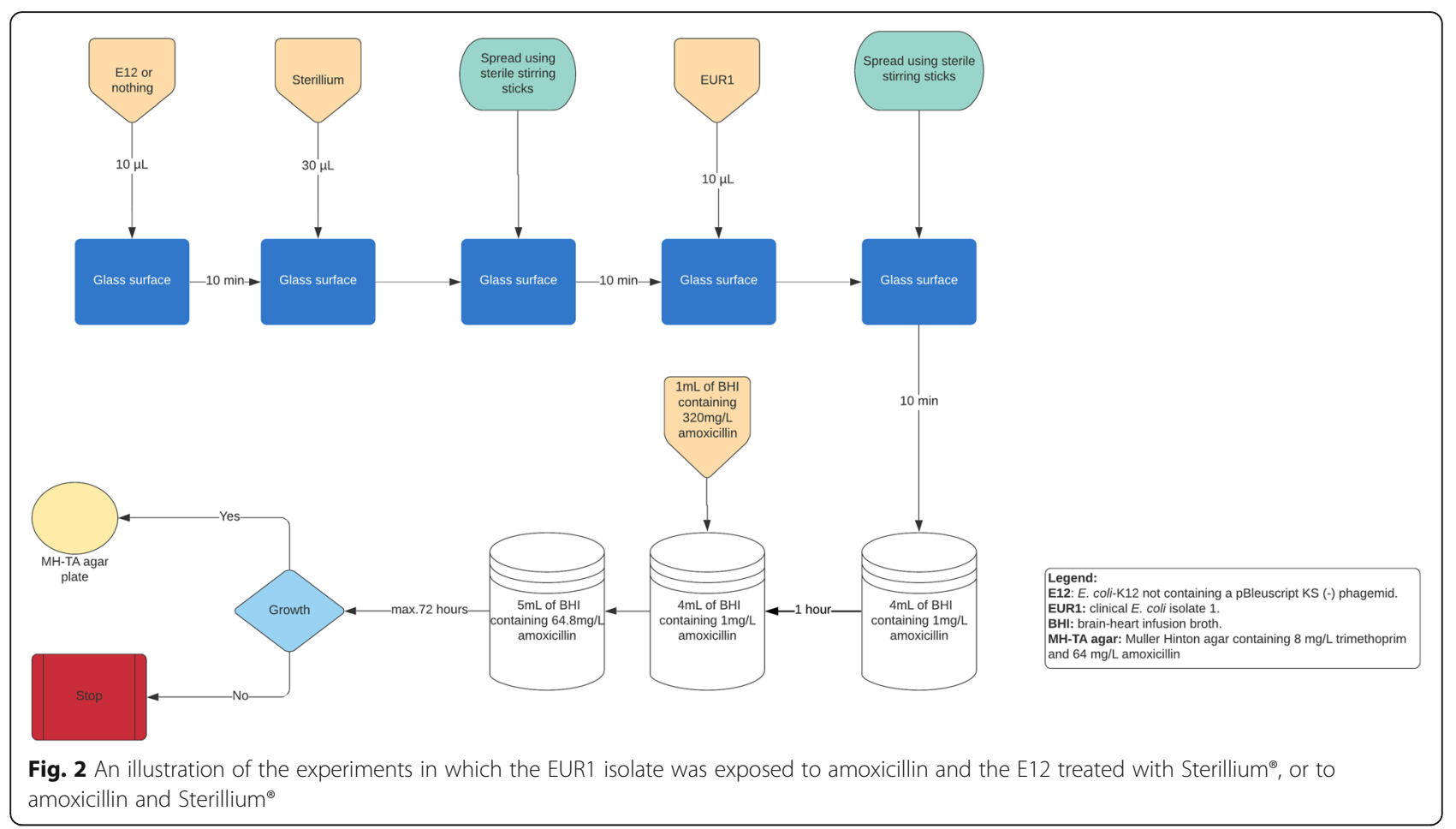

glasses were placed in a container with $5 \mathrm{~mL}$ of BHI, vortexed for $30 \mathrm{~s}$ at 2800 rotations per minute and incubated for $72 \mathrm{~h}$ at 35 to $37 \mathrm{C}^{\circ}$, visually inspecting growth at 24,48 and $72 \mathrm{~h}$. In case there was no visible growth after $72 \mathrm{~h}$ of incubation, $1 \mu \mathrm{L}$ of broth was plated on Muller Hinton agar.

\section{Whole-genome shotgun sequencing (WGS) and de novo assembly}

The wildtype isolates (EUR1, EUR2), the pBS-E12 and E12 strains, and a selection of the mutant isolates were sequenced on an Illumina MiSeq (Illumina, San Diego, United States) and assembled with CLC Genomics Workbench v.11 or v.12 (Qiagen, Hilden, Germany). Selection criteria were: in the first thirteen experiments in which growth of a mutant isolate was detected, an isolate was sent for sequencing. The following quality control criteria were used: coverage $\geq 20$; number of scaffolds $\leq 1000$; N50 $\geq 15.000$ bases and maximum scaffold length $\geq 50.000$ bases.

\section{Mechanism of resistance}

The presence of genomic resistance determinants conferring amoxicillin and trimethoprim resistance in the assembled genomes of the mutated E. coli was identified with the online bioinformatic tools ResFinder v3.1 and PointFinder v3.1 (Center for Genomic Epidemiology, Technical University of Denmark, Lingby, Denmark) [28, 29]. Genomes were screened for known and unknown chromosomal resistance mutations.
Acquired resistance genes were called when at least $60 \%$ of the length of the best matching gene in the ResFinder database was covered with a sequence identity of at least $90 \%$. If an unknown or known chromosomal mutation was detected in genomic regions implicated in betalactam resistance using PointFinder, the region was extracted from the assembled genome using Biopython v.1.73. Subsequently, the extracted genomic regions were aligned using Vector NTI Advance 11 software (ThermoFisher Scientific, Waltham, USA) to the corresponding region of either the EUR1 or EUR2 isolate and the corresponding region of the E. coli $\mathrm{K}-12$ strain MG1655 (GenBank database accession number NC000913.3), the pBS-E12 strain, and the E12 strain. Moreover, to verify that the observed amoxicillin resistance was not the result of the acquisition of the amoxicillin resistance gene from the pBleuscript(-) phagemid, all assembled genomes were screened for the presence of this gene using ABRicate v.0.8.13 with the same coverage and identity thresholds as those used in ResFinder. Additionally, all mutated strains and the pBS-E12, EUR1, EUR2 isolates were phenotypically screened for AmpC production using the D68C AmpC \& ESBL Detection set (Mastdiscs, Mastgroup Ltd., Bootle United Kingdom) and interpreted according to manufacturer's instruction.

\section{Whole-genome multilocus sequence typing}

Whole-genome multilocus sequence typing (wgMLST) (core and accessory genome) was performed of both wildtype isolates and all sequenced mutant isolates using 
Ridom SeqSphere+, version 5.1.0. (Ridom, Münster, Germany). Species-specific typing schemes were used as described by Kluytmans-Van den Bergh et al. [30]. Allto-all pairwise genetic difference was calculated between the isolates by counting the total number of allele differences in the wgMLST typing scheme and by dividing the total number of allele differences in the wgMLST typing scheme by the total number of shared alleles in the wgMLST typing scheme, ignoring pairwise missing values.

\section{Statistics}

Risk differences were estimated using a generalised linear model with binomial distribution, an identity link and robust error estimation (SPSS version 25).

\section{Accession numbers}

All generated raw reads were submitted to the European Nucleotide Archive (ENA) of the European Bioinformatics Institute (EBI) under the study accession number: PRJEB34354.

\section{Results}

\section{Resistance induction experiment}

In $22(11.0 \%)$ of 200 experiments in which the EUR1 strain was exposed to the remnants of the pBS-E12 and Sterillium $^{\circ}$ an amoxicillin- and trimethoprim-resistant $E$. coli isolate (EUR1M1 - EUR1M22) was obtained, as opposed to only 2 (1.0\%) (EUR1S1, EUR1S2) of 200 experiments involving the exposure of the EUR1 strain to Sterillium ${ }^{\circ}$ only (risk difference $10.0 \%$; $95 \%$ CI $5.4-$ $14.6 \%)$, and 1 (0.5\%) (EUR1E1) of 200 experiments involving the exposure of the EUR1 strain to the remnants of the E12 and Sterillium (risk difference 10.5\%; 95\% CI 6.1-14.9\%). In the experiments performed using the EUR2, 1 mutant isolate (EUR2M1) was grown in 48 experiments. Amoxicillin-resistant strains did not only show increased MICs for amoxicillin but also for amoxicillin-clavulanic acid, piperacillin-tazobactam or cephalosporins (Table 1). No increases in the MIC were observed for carbapenems and non-beta-lactam antibiotics tested. One out of 100 disinfection experiments showed growth of the pBS-E12, indicating that in only a minimal number of experiments the amoxicillin concentration during the experiments was influenced by viable beta-lactamase-producing pBS-E12.

\section{Mechanisms of amoxicillin resistance}

A selection of the isolates was sequenced, i.e. 10 mutant E. coli isolates from the experiment in which EUR1 was exposed to the remnants of the pBS-E12, 2 mutants from the control experiments, 1 mutant from the experiments with the EUR2 isolate, the wildtype isolates EUR1 and EUR2, and the pBS-E12 and E12 strains. Table 2 shows the various mutations at given positions of the promoter/attenuator region of the cAmpC gene for every sequenced isolate in this study. Known and unknown mutations in the promoter/attenuator region of the chromosomal AmpC beta-lactamase (cAmpC) gene were detected in the genomes of all mutant $E$. coli isolates that were sequenced (EUR1M1-EUR1M10, EUR1S1-EUR1S2, EUR2M1), but not in the EUR1 isolate. Two unknown mutations were detected in the promoter/attenuator region of the EUR2 isolate (Table 2). Alignment against the corresponding region of an $E$. coli $\mathrm{K}-12$ strain revealed that one of these mutations was in the alternate -10 box promoter box (Table 2). However, these mutations did not increase the MIC for amoxicillin in the EUR2 isolate. In every mutant (EUR1M1-EUR1M10, EUR1S1-EUR1S2, EUR2M1) alignment of the cAmpC promoter/attenuator region revealed mutations, when compared to the same genomic region of the corresponding wildtype isolates, in regions implicated in cAmpC hyperproduction as described by Tracz et al. (Table 2) [31]. Moreover, the different promoter/attenuator regions present in the mutant EUR1 isolates (Table 2) suggests de novo mutation rather than the selection of a previously present mutated subpopulation. No mutations in other regions implicated in beta-lactam resistance were detected, nor were acquired beta-lactam resistance genes detected in any of the mutated (EUR1M1-EUR1M10, EUR1S1-EUR1S2, EUR2M1) or wildtype isolates (EUR1, EUR2). The amoxicillin-resistance gene of the pBleuscript $\mathrm{KS}(-)$ phagemid was not detected in any of the sequenced isolates except the pBS-E12. In the pBS-E12 and E12 strain alignment of the cAmpC promotor/attenuator did not reveal mutations, when compared to the same genomic region of the E. coli $\mathrm{K}-12$ strain MG1655. A dfrA1 gene conferring trimethoprim resistance was detected in the EUR1, EUR2 and all sequenced mutant isolates but not in the pBS-E12 and E12 strains.

Phenotypic tests for AmpC beta-lactamase production were negative for the two wildtype isolates and the pBSE12 isolate. However, for all mutants, phenotypic testing showed AmpC beta-lactamase production (Table 1; Supplementary Table S3).

\section{Whole-genome multilocus sequence typing}

The number of allele differences between the mutated EUR1M1-EUR1M10 isolates and wildtype EUR1 isolate ranged from $21(0.59 \%)$ to 69 (1.96\%) (median: 44.5 (1.27\%)). In all but one (EUR1M9) of the mutant EUR1 isolates, the difference between the mutant and corresponding control exceeded the threshold for genetic distance between related and unrelated isolates as defined by Klutymans-van den Bergh et al. (Fig. 3) [30]. Both mutated isolates from the control experiment (EUR1S1 
Table 2 Mutations in CAmpC promoter/attenuator region of the wildtype and mutant isolates

\begin{tabular}{|c|c|c|c|c|c|c|c|c|c|c|}
\hline \multirow{3}{*}{$\begin{array}{l}\text { E.coli K-12 } \\
\text { (wildtype) }\end{array}$} & \multicolumn{9}{|c|}{ Base in wildtype $c A m p C$ promoter/attenuator region } & \multirow{5}{*}{$\begin{array}{l}\text { Date } \\
\text { experiments } \\
\text { performed }^{\text {a }}\end{array}$} \\
\hline & $\overline{-32}$ & -23.1 & -21.1 & -19 & -18 & -16.1 & -11 & +58 & $\overline{+81}$ & \\
\hline & $\mathrm{T}$ & - & - & $\mathrm{T}$ & G & - & C & C & G & \\
\hline \multirow[t]{2}{*}{ Isolate } & \multicolumn{9}{|c|}{ Position of mutation in cAmpC promoter/attenuator region ${ }^{a}$} & \\
\hline & -32 & -23.1 & -21.1 & -19 & -18 & -16.1 & -11 & +58 & +81 & \\
\hline \multicolumn{11}{|l|}{$\overline{\mathrm{pBS}-\mathrm{E} 12^{¥}}$} \\
\hline \multicolumn{11}{|l|}{ E12 } \\
\hline EUR1 (wildtype) & & & & & & & & & A & \\
\hline EUR1M1 & A & & & & & & & & A & 10th May \\
\hline EUR1M2 & & G & C & A & & & & & A & 10th May \\
\hline EUR1M3 & & G & C & A & & & & & A & 11th May \\
\hline EUR1M4 & & G & C & A & & & & & A & 12th May \\
\hline EUR1M5 & & G & C & A & & & & & A & 12th May \\
\hline EUR1M6 & & & & & & & $\mathrm{T}$ & & A & 13th May \\
\hline EUR1M7 & & G & C & A & & & & & A & 25th May \\
\hline EUR1M8 & & G & C & A & & & & & A & 26th May \\
\hline EUR1M9 & & G & C & A & & & & & A & 1st June \\
\hline EUR1M10 & & & & & & & $\mathrm{T}$ & & A & 1st June \\
\hline EUR1S1 & & & $\mathrm{T}$ & & & & & & A & 23th May \\
\hline EUR1S2 & A & & & & & & & & A & 17th August \\
\hline EUR2 (wildtype) & & & & & A & & & $\mathrm{T}$ & & \\
\hline EUR2M1 & & & & & A & A & & $\mathrm{T}$ & & 12th May \\
\hline
\end{tabular}

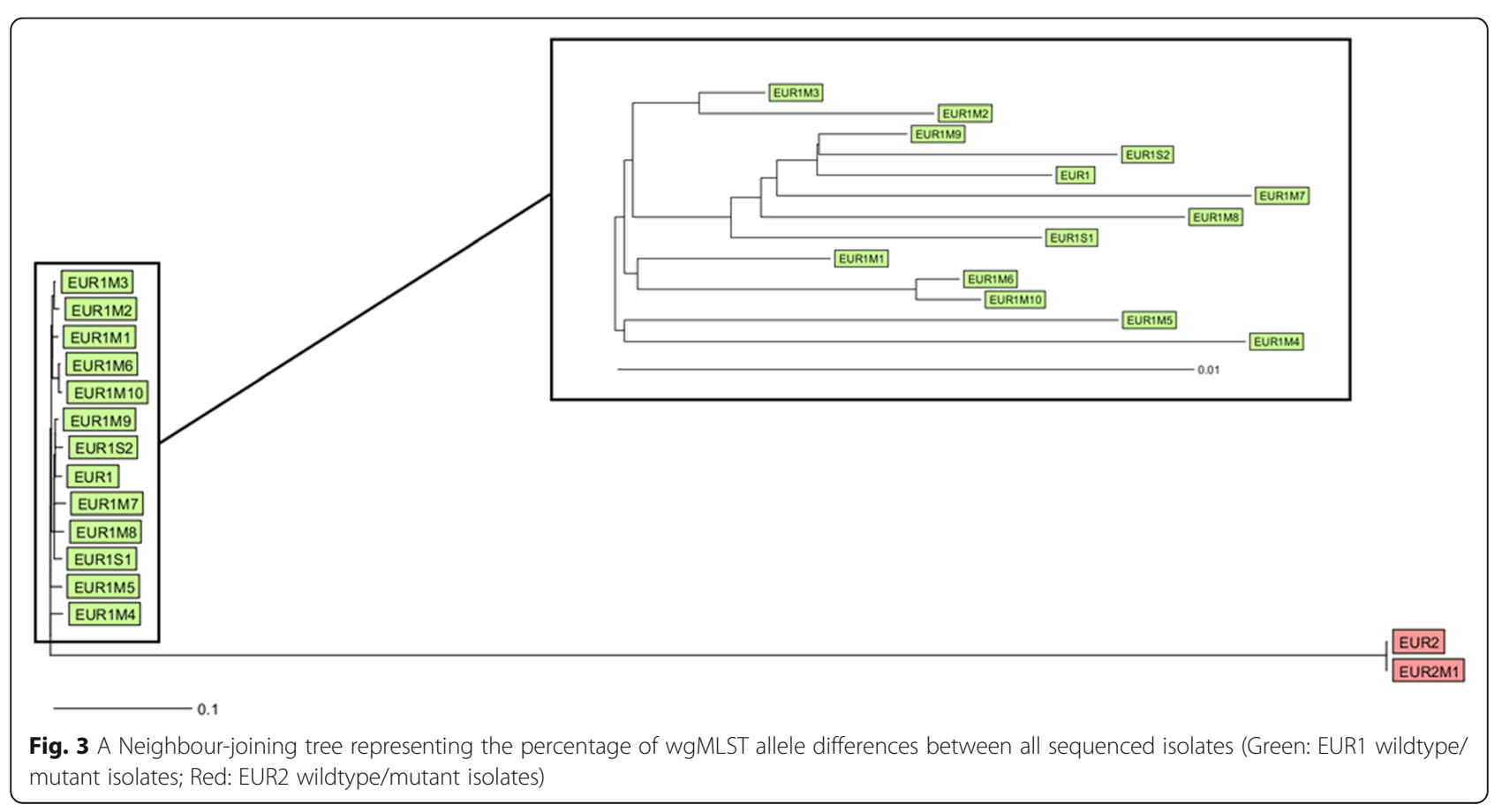


and EUR1S2) also showed a high number of allele differences when compared to the EUR1 wildtype (EUR1S1: $n=34(0.96 \%)$, EUR1S2: $n=36(1.02 \%))$. The high number of allele differences between the various mutated EUR1 isolates suggests de novo mutation acquisition rather than the selection of a previously present mutated subpopulation (Fig. 3). In the EUR2M1, when compared to its wildtype, no allele differences were observed. The number of allele differences between the EUR1 and EUR2 isolate was 3184 (97.73\%)(Fig. 3).

\section{Discussion}

An in vitro model was developed to simulate propanolbased hand disinfection as is common practice in healthcare facilities nowadays. Bacteria are effectively killed, but their remnants remain present after disinfection. Subsequently susceptible bacteria are exposed to the remaining debris, including DNA. Experiments performed using this model showed a significant increase in the development of amoxicillin resistance in E. coli isolates after exposure to the remnants of the pBS-E12 treated with a propanol-based hand rub as compared to exposure to remnants of the E-12 treated with a propanol-based hand rub and as compared to exposure to only a propanol-based hand rub. AmpC betalactamase hyperproduction, due to mutations in the AmpC promoter/attenuator region, was responsible for the development of the observed AMR. The number of resistant mutants that developed in the isolates exposed to the remnants of the pBS-E12 also exceeded the number of pBS-E12 growing in the disinfection experiments, which indicates that amoxicillin degradation by the pBSE12 beta-lactamase was not responsible for the observed increase in AMR. All mutated EUR1 isolates showed a high number of allele differences when compared to the wildtype isolate, suggesting genome-wide mutations. Interestingly this genome-wide mutational pattern was not detected in the EUR2 isolate. Indicating that the genome-wide mutational pattern might not only be (control) experiment but also isolate related.

The exact underlying mechanism leading to the AMR conferring mutations in the promoter/attenuator region of the cAmpC remains unknown. Since the amoxicillin resistance gene of the pBluescript phagemid was not detected in the EUR1 and EUR2 mutant isolates, it seems more likely that the remnants of the pBS-E12 increased the mutation rate in the EUR1 and EUR2 isolate facilitating AMR development through cAmpC hyperproduction. Previous studies have implicated several mechanisms that may increase the mutation rate and result in AMR, such as the general stress response and an increased constitutive mutation frequency [32-35]. Further studies are needed to evaluate the role of these mechanisms in our experiments. Moreover, mutagenic assays with other antibiotics (e.g. streptomycin and nalidixic acid) need to be performed, to further assess the mutagenic potential of the remnants of the pBS-E12 and Sterillium ${ }^{\circ}$ in the EUR1 isolate. However, the remnants of the pBS-E12 could also facilitate the development of AMR by enabling the EUR1 and EUR2 isolates to survive longer under high amoxicillin concentrations, possibly through providing nutrients or through efflux pump activation, thereby increasing the chance of the occurrence of a mutation leading to $\mathrm{cAmpC}$ hyperproduction. A small degree of heterogeneity in the $\mathrm{cAmpC}$ promotor region of the $\mathrm{pBS}-\mathrm{E} 12$ population that lead to $\mathrm{cAmpC}$ hyperproduction cannot be completely ruled out. Therefore, it still remains possible that the $\mathrm{cAmpC}$ promotor region of this heterogeneous subpopulation was horizontally transferred to the wildtype EUR1 and EUR2 isolates.

Several studies have shown that environmental stressors other than antibiotics can influence the development of AMR [35-38]. However, the association between exposure to remnants of phagemid-carrying $E$. coli isolates and increased development of AMR has not been investigated to date. Interestingly, this increase in the development of AMR was only related to exposure to remnants of phagemid-containing $E$. coli isolates. Other studies have already shown that extracellular DNA can lead to the development of AMR in other bacteria [22, 23]. However, this is the first study relating exposure to external DNA to the development of beta-lactam resistance through chromosomal mutations in E. coli.

Despite the increased development of AMR was only related to exposure to remnants of the pBS-E12, it remains unknown if and what specific compounds of Sterillium ${ }^{\circ}$ contribute to the observed increased development of AMR. Contrary to other alcohol-based hand rubs, Sterillium ${ }^{\bullet}$ contains mecetronium etilsulfate which potentially has a lasting antimicrobial effect [39, 40]. Further studies are needed to evaluate if this increased development of AMR also occurs with other alcoholbased hand rubs not containing such compounds. Furthermore, the current study only simulates hand disinfection procedures so it remains unknown to what extent this phenomenon also could apply to environmental disinfection.

Although cAmpC hyperproduction has been implicated in beta-lactam resistance in $E$. coli isolates following exposure to stepwise increasing concentrations of amoxicillin over several days [41, 42]. cAmpC hyperproduction in this study occurred after only a short time of exposure to amoxicillin concentrations lower than the MIC of the exposed E. coli isolate. Even in our control experiments, in which the $E$. coli isolates were only exposed to Sterillium ${ }^{\circ}$, cAmpC hyperproduction developed 
after only short sub-inhibitory amoxicillin concentrations. Moreover, in the isolates EUR1M2-EUR1M5 and EUR1M7-9 three mutations were detected in the cAmpC promotor region. Each of these mutations individually could increase cAmpC production in $E$. coli $[31,43]$. Perhaps, consecutive mutations, not a single mutational event during the short time of sub-inhibitory amoxicillin concentration, lead to a step-wise increase in amoxicillin MIC in these mutant isolates. In the study by Kohanski et al. [44] exposure to sub-lethal levels of amoxicillin also resulted in MIC increases to antimicrobials other than from the beta-lactam group. We did not observe MIC increases or known mutations in resistance-associated genes of antibiotics other than the beta-lactams.

Contrary to other studies investigating the development of AMR in $E$. coli $[41,44]$, we used two clinical $E$. coli isolates for amoxicillin resistance induction. Moreover, our in vitro model simulates propanol-based hand disinfection procedures which are very common in clinical practice $[4,5,45]$. Also, the duration of exposure to sub-inhibitory amoxicillin concentrations closely reflects the duration of sub-inhibitory amoxicillin plasma concentrations in patients at the start of amoxicillin treatment [27].

This study has some limitations. The pBluescript-KS $(-)$ phagemid is a cloning vector not present in clinical isolates. Whether remnants of $E$. coli isolates containing wildtype phagemids also increase the development of AMR needs further investigation. Moreover, since the pBluescript KS(-) phagemid contains both a bacteriophage origin of replication and a plasmid origin of replication future studies are required to assess whether the observed effect is bacteriophage or plasmid related. Also, only a limited number of isolates were used in this study. The extent to which this AMR induction is possible in other $E$. coli isolates or other species remains unknown. Moreover, it remains unknown whether and to what extent this in vitro phenomenon plays a role in the development of AMR in vivo.

To the best of our knowledge, this is the first study showing development of amoxicillin resistance in an $E$. coli isolate after exposure to a phagemid-containing $E$. coli treated with a propanol-based hand rub.

\section{Conclusion}

This exploratory study showed the development of amoxicillin resistance in an E. coli isolate after exposure to an unrelated phagemid-containing $E$. coli treated with a propanol-based hand rub. Although phagemids are cloning vectors that are not present in clinical isolates, this finding may have implications for hand disinfection practices in healthcare facilities.

\section{Supplementary information}

Supplementary information accompanies this paper at https://doi.org/10. 1186/s13756-020-00708-7.

Additional file 1: Table S1. Characteristics of used strains. Table S2. Features of pBleuscript KS(-) phagmid ATCC ${ }^{\oplus} 87047$ TM. Table S3. Inhibition zone diameters of D68C AmpC \& ESBL detection set for the pBS-E12, EUR1, EUR2 and mutant isolates.

\section{Abbreviations}

AMR: Antimicrobial resistance; pBS-E12: E. coli -K12 containing a pBleuscript KS (-) phagemid encoding for an amoxicillin resistance gene (bla $\left.a_{\mathrm{TEM}-116}\right)$; EUR1: Clinical E. coli isolate 1; EUR2: Clinical E. coli isolate 2; MIC: Minimal inhibitory concentration; BHI: Brain-heart infusion broth; MH-TA: Muller Hinton agar containing $8 \mathrm{mg} / \mathrm{L}$ trimethoprim and $64 \mathrm{mg} / \mathrm{L}$ amoxicillin; WGS: Whole genome shotgun sequencing; ENA: European Nucleotide Archive; EBl: European Bioinformatics Institute; cAmpC: chromosomal AmpC beta-lactamase

\section{Acknowledgments}

We kindly thank the Avans university of applied sciences Breda for providing the JM83, JM109 isolates and pBluescript KS(-) phagemid.

\section{Authors' contributions \\ JS performed the experiments, analysed and interpreted the data, and drafted the manuscript. MK analysed and interpreted the data, and revised the manuscript. CV performed the experiments, and revised the manuscript. $\mathrm{JR}$ analysed and interpreted the data, and revised the manuscript. JK analysed and interpreted the data, and revised the manuscript. All authors read and approved the final manuscript.}

\section{Funding}

None

Availability of data and materials

The datasets supporting the conclusions of this article is included within the article and its additional file.

Ethics approval and consent to participate

Not applicable.

\section{Consent for publication}

Not applicable.

\section{Competing interests}

The authors declare that they have no competing interests.

\section{Author details}

${ }^{1}$ Department of Infection Control, Amphia Hospital, Breda, the Netherlands. ${ }^{2}$ Laboratory for Medical Microbiology and Immunology,

Elisabeth-TweeSteden Hospital, Tilburg, the Netherlands. ${ }^{3}$ Amphia Academy Infectious Disease Foundation, Amphia Hospital, Breda, the Netherlands.

${ }^{4}$ Julius Center for Health Sciences and Primary Care, University Medical Center Utrecht, Utrecht University, Utrecht, the Netherlands. ${ }^{5}$ Department of Medical Microbiology and Infection Prevention, University of Groningen, University Medical Center Groningen, Groningen, the Netherlands.

Received: 29 October 2019 Accepted: 11 March 2020

Published online: 16 March 2020

References

1. Bonnet R. Growing group of extended spectrum: the CTX-M enzymes. Antimicrob Agent Chemother. 2004;48(1):1-14.

2. Nordmann P, Naas T, Poirel L. Global spread of Carbapenemase producing Enterobacteriaceae. Emerg Infect Dis. 2011;17(10):1791-8.

3. Mathers AJ, Peirano G, Pitout JDD. The role of epidemic resistance plasmids and international high- risk clones in the spread of multidrug-resistant Enterobacteriaceae. Clin Microbiol Rev. 2015;28(3):565-91.

4. Tacconelli E, Cataldo MA, Dancer SJ, De Angelis G, Falcone M, Frank U, et al. ESCMID guidelines for the management of the infection control measures 
to reduce transmission of multidrug-resistant gram-negative bacteria in hospitalized patients. Clin Microbiol Infect. 2014;20(s1):1-55.

5. Boyce J, Pittet D. Centers for Disease Control and Prevention. Guideline for hand hygiene in health-care settings: recommendations of the healthcare infection control practices advisory committee and the HICPAC/SHEA/APIC/ IDSA hand hygiene task force. MMWR Recomm Rep. 2002;51:1-45.

6. Kampf G, Ostermeyer C, Heeg P, Paulson D. Evaluation of two methods of determining the efficacies of two alcohol-based hand rubs for surgical hand antisepsis. Appl Environ Microbiol. 2006;72(6):3856-61.

7. Kampf G, Kapella M. Suitability of Sterillium gel ${ }^{\mathrm{TM}}$ for surgical hand disinfection. J Hosp Infect. 2003;54(3):222-5.

8. Kampf G, Ostermeyer C, Heeg P. Surgical hand disinfection with a propanolbased hand rub: equivalence of shorter application times. J Hosp Infect. 2005;59(4):304-10.

9. Kramer A, Rudolph P, Kampf G, Pittet D. Limited efficacy of alcohol-based hand gels. Lancet. 2002;359(9316):1489-90.

10. Mao D, Luo Y, Mathieu J, Wang Q, Feng L, Mu Q, et al. Persistence of extracellular DNA in river sediment facilitates antibiotic resistance gene propagation. Environ Sci Technol. 2014;48(1):71-8.

11. Nielsen KM, Smalla K, Van Elsas JD. Natural transformation of Acinetobacter sp. strain BD413 with cell lysates of Acinetobacter sp., Pseudomonas fluorescens, and Burkholderia cepacia in soil microcosms. Appl Environ Microbiol. 2000;66(1):206-12.

12. Kwon DH, Dore MP, Kim JJ, Kato M, Lee M, Wu JY, et al. High-level $\beta$-lactam resistance associated with acquired multidrug resistance in Helicobacter pylori. Antimicrob Agents Chemother. 2003;47(7):2169-78.

13. Bae J, Oh E, Jeon B. Enhanced transmission of antibiotic resistance in Campylobacter jejuni biofilms by natural transformation. Antimicrob Agents Chemother. 2014;58(12):7573-5.

14. Trzciński K, Thompson CM, Lipsitch M. Single-step capsular transformation and acquisition of penicillin resistance in Streptococcus pneumoniae. J Bacteriol. 2004;186(11):3447-52.

15. Johnston C, Martin B, Fichant G, Polard P, Claverys J-P. Bacterial transformation: distribution, shared mechanisms and divergent control. Nat Rev Microbiol. 2014;12(3):181-96.

16. Domingues S, Harms K, Fricke WF, Johnsen PJ, da Silva GJ, Nielsen KM Natural Transformation Facilitates Transfer of Transposons, Integrons and Gene Cassettes between Bacterial Species. PLoS Pathog. 2012;8(8):e1002837.

17. Sun D, Zhang $Y$, Mei $Y$, Jiang $H$, Xie Z, Liu H, et al. Escherichia coli is naturally transformable in a novel transformation system. FEMS Microbiol Lett. 2006; 265(2):249-55.

18. Baur B, Hanselmann K, Schlimme W, Jenni B. Genetic transformation in freshwater: Escherichia coli is able to develop natural competence. Appl Environ Microbiol. 1996;62(10):3673-8.

19. Maeda S, Ito M, Ando T, Ishimoto $Y$, Fujisawa $Y$, Takahashi $H$, et al. Horizontal transfer of nonconjugative plasmids ina colony biofilm of Escherichia coli. FEMS Microbiol Lett. 2006;255(1):115-20.

20. Maeda S, Sawamura A, Matsuda A. Transformation of colonial Escherichia coli on solid media. FEMS Microbiol Lett. 2004;236(1):61-4.

21. Etchuuya R, Ito M, Kitano S, Shigi F, Sobue R, Maeda S. Cell-to-cell transformation in Escherichia coli: a novel type of natural transformation involving cell-derived DNA and a putative promoting pheromone. PLoS One. 2011;6(1):e16355.

22. Johnson L, Horsman SR, Charron-Mazenod L, Turnbull AL, Mulcahy H, Surette MG, et al. Extracellular DNA-induced antimicrobial peptide resistance in Salmonella enterica serovar Typhimurium. BMC Microbiol. 2013;13(1):1 Available from: BMC Microbiology.

23. Lewenza S. Extracellular DNA-induced antimicrobial peptide resistance mechanisms in Pseudomonas aeruginosa. Front Microbiol. 2013;4(FEB):1-6.

24. The European Committee on Antimicrobial Susceptibility Testing. Breakpoint tables for interpretation of MICs and zone diameters. Version 8.1, 2018. http://www.eucast.org.

25. Lee J, Choi J, Kim H. Determination of hand surface area by sex and body shape using alginate. J Physiol Anthropol. 2007;26(4):475-83.

26. Girard R, Aupee M, Erb M, Bettinger A, Jouve A. Hand rub dose needed for a single disinfection varies according to product: a bias in benchmarking using indirect hand hygiene indicator. J Epidemiol Glob Health. 2012;2(4):193-8.

27. de Velde F, de Winter BCM, Koch BCP, van Gelder T, Mouton JW. Non-linear absorption pharmacokinetics of amoxicillin: consequences for dosing regimens and clinical breakpoints. J Antimicrob Chemother. 2016;71(10): 2909-17.
28. Zankari E, Allesøe R, Joensen KG, Cavaco LM, Lund O, Aarestrup FM PointFinder: a novel web tool for WGS-based detection of antimicrobial resistance associated with chromosomal point mutations in bacterial pathogens. J Antimicrob Chemother. 2017;72(10):2764-8.

29. Zankari E, Hasman H, Cosentino S, Vestergaard M, Rasmussen S, Lund O, et al. Identification of acquired antimicrobial resistance genes. J Antimicrob Chemother. 2012;67(11):2640-4.

30. Kluytmans-van den Bergh MFQ, Rossen JWA, Bruijning-Verhagen PCJ, Bonten MJM, Friedrich AW, Vandenbroucke-Grauls CMJE, et al. Wholegenome multilocus sequence typing of extended-Spectrum-Beta-lactamaseproducing Enterobacteriaceae. J Clin Microbiol. 2016;54(12):2919-27.

31. Tracz DM, Boyd DA, Hizon R, Bryce E, McGeer A, Ofner-Agostini M, et al. ampC gene expression in promoter mutants of cefoxitin-resistant Escherichia coli clinical isolates. FEMS Microbiol Lett. 2007;270(2):265-71.

32. Firmino N, Goldsmith J-M, Morin A. Ampicillin resistance is increased in Escherichia coli $\mathrm{K} 12$ relA and spoT mutants but sub-inhibitory pretreatment does not induce adaptive resistance. J Exp Microbiol Immunol. 2013; 17(April):34-9.

33. Baharoglu Z, Mazel D. Vibrio cholerae triggers SOS and mutagenesis in response to a wide range of antibiotics: a route towards multiresistance. Antimicrob Agents Chemother. 2011;55(5):2438-41.

34. Eliopoulos GM, Blazquez J. Hypermutation as a factor contributing to the acquisition of antimicrobial resistance. Clin Infect Dis. 2003;37(9):1201-9.

35. Petrosino JF, Galhardo RS, Morales LD, Rosenberg SM. Stress-induced $\beta$ lactam antibiotic resistance mutation and sequences of stationary-phase mutations in the Escherichia coli chromosome. J Bacteriol. 2009;191(19): 5881-9.

36. Fruci M, Poole K. Bacterial stress responses as determinants of antimicrobial resistance. In: Stress and environmental regulation of gene expression and adaptation in Bacteria. Hoboken: Wiley; 2016. p. 115-36.

37. Nishino K, Yamasaki S, Hayashi-Nishino M, Yamaguchi A. Effect of NlpE overproduction on multidrug resistance in Escherichia coli. Antimicrob Agents Chemother. 2010;54(5):2239-43.

38. Raivio TL, Leblanc SKD, Price NL. The Escherichia coli Cpx envelope stress response regulates genes of diverse function that impact antibiotic resistance and membrane integrity. J Bacteriol. 2013;195(12):2755-67.

39. Kampf G. Lack of antimicrobial efficacy of mecetronium etilsulfate in propanol-based hand rubs for surgical hand disinfection. J Hosp Infect. 2017;96(2):189-91.

40. Kampf G, Kramer A, Suchomel M. Lack of sustained efficacy for alcoholbased surgical hand rubs containing 'residual active ingredients' according to EN 12791. J Hosp Infect. 2017;95(2):163-8.

41. van der Horst MA, Schuurmans JM, Smid MC, Koenders BB, ter Kuile BH. De novo Acquisition of Resistance to three antibiotics by Escherichia coli. Microb Drug Resist. 2011;17(2):141-7.

42. Händel N, Schuurmans JM, Feng Y, Brul S, ter Kuile BH. Interaction between mutations and regulation of gene expression during development of De novo antibiotic resistance. Antimicrob Agents Chemother. 2014;58(8):4371-9.

43. Mulvey MR, Bryce E, Boyd DA, Ofner-Agostini M, Land AM, Simor AE, et al. Molecular characterization of cefoxitin-resistant Escherichia coli from Canadian hospitals. Antimicrob Agents Chemother. 2005;49(1):358-65.

44. Kohanski MA, DePristo MA, Collins JJ. Sublethal antibiotic treatment leads to multidrug resistance via radical-induced mutagenesis. Mol Cell. 2010;37(3): 311-20.

45. Rutala WA, Weber DJ. Disinfection and sterilization in health care facilities: what clinicians need to know. Clin Infect Dis. 2004;39(5):702-9.

\section{Publisher's Note}

Springer Nature remains neutral with regard to jurisdictional claims in published maps and institutional affiliations. 